\title{
Ortopedia regenerativa durante la contingencia COVID-19
}

\author{
Regenerative orthopedics during the COVID-19 contingency \\ José Fernando Izquierdo Moncayo, * José Luis Carrillo Gamboa ${ }^{\ddagger}$ \\ * Especialista en Traumatología y Ortopedia. Miembro Comité Científico FEMECOT. \\ Hospital Ángeles Tijuana. Tijuana, B.C. \\ ‡ Especialista en Traumatología y Ortopedia. Médica Tec100. Director \\ Centro Ortopedia Regenerativa, CORE. Querétaro, Qro.
}

Correspondencia: Dr. José Fernando Izquierdo Moncayo. Torre de Consultorios Hospital Ángeles Tijuana. Paseo de los Héroes No. 10999 Interior 309, Zona Urbana Río, 22010, Tijuana, B.C. Correo electrónico: fdoizquierdo728@gmail.com

\section{RESUMEN}

Los tratamientos ortobiológicos se llevan a cabo en tres escenarios: 1) consultorio, 2) gabinete de rayos $X$ y 3) quirófano. Los que se realizan en el consultorio son alrededor de $70 \%$, incluyendo aquellos que se hacen guiados por ultrasonido. Todos son procedimientos ambulatorios o de corta estancia (menor de cuatro horas). En lo posible se deben posponer todos los procedimientos de ortopedia regenerativa durante la contingencia por COVID-19. Podrán ser tratados aquellos pacientes que no responden al manejo conservador o que requieren de algún procedimiento y que se necesite "comprar tiempo a la cirugía». El procedimiento se debe hacer en el consultorio o si se requiere de fluoroscopio en una clínica de corta estancia. Tomando todas las precauciones ya mencionadas en las guías de manejo de pacientes con sospecha de COVID-19, todo paciente deberá, de ser posible, hacerse la prueba tres días previos a su procedimiento. Sólo se realizarán aquellos procedimientos que sean bajo anestesia local/ sedación o sin anestesia. No se efectuará ningún procedimiento que requiera de intubación. Por lo anterior, consideramos que $95 \%$ de los procedimientos pueden ser pospuestos. La consulta de seguimiento deberá ser cada cuatro u ocho semanas o mediante telemedicina.

Palabras clave: Ortopedia regenerativa, procedimientos, diferir, no intubación, uso equipo de protección personal.

\section{ABSTRACT}

Orthobiologic treatments can be done in 3 settings: 1) medical office, 2) $X$-ray department, 3) operating room. In office procedures account for $70 \%$ of total, including those done under ultrasound guidance. All procedures should be done in an outpatient setting (less than four hours) if possible, all procedures must be postponed until the COVID-19 restrictions are lifted. Patients that do not respond to conservative treatment or need a regenerative procedure to «buy time» for an elective surgery can be done following all the safety measures included in COVID-19 surgical guidelines. The procedure should be done at a medical office or at an ambulatory surgery center, especially if fluoroscopy is needed. You should only do those procedures that can be done under local/ sedation anesthesia. Do not do any patient that can require intubation! We consider that $95 \%$ of all orthobiologic procedures can be postponed: follow up should be done every four to eight weeks or by telemedicine.

Keywords: Regenerative orthopedics, procedures, postpone, no intubation, personal protection equipment use. 


\section{ORTOPEDIA REGENERATIVA. ¿QUÉ DEBO HACER?}

Si el año pasado nos hubiesen anticipado que el mundo se detendría, que estaríamos en medio de una pandemia, viviendo medidas de distanciamiento social para tratar de disminuir lo más posible las pérdidas humanas, difícilmente lo hubiésemos creído. Sin embargo, aquí estamos llenos de preguntas y de incertidumbre. Nadie nos preparó para esta pandemia y nos tomó sin tener todas las herramientas, pero debemos hacer nuestro trabajo de la mejor manera posible. Iniciemos por decir qué es la ortopedia regenerativa, es una rama emergente de la medicina regenerativa, que a su vez es una rama de la bioingeniería. Se compone de una combinación de células, métodos de ingeniería de materiales y bioquímica para mejorar o reemplazar funciones biológicas. La ortopedia regenerativa es un campo amplio que incluye la ingeniería de tejidos, pero también incorpora la investigación sobre la autocuración, donde el cuerpo usa sus propios sistemas para reconstruir tejidos y recrear células. No es una cura milagrosa, sólo es una herramienta terapéutica adicional a los tratamientos tradicionales. Puede ser considerada un tratamiento «puente» entre el manejo conservador y los tratamientos quirúrgicos o definitivos. La ortopedia regenerativa tiene como retos estimular la regeneración de los tejidos, reparar lesiones musculares, de cartílago, tendones, ligamentos y hueso así como ralentizar el envejecimiento. Cuando se produce una lesión, el tejido de cicatriz llena los defectos y restablece la continuidad morfológica, pero lo hace a base de tejido conectivo que sustituye las células especializadas y que carece de función. Los tratamientos ortobiológicos van a lograr la reparación mediante la sustitución de células muertas o dañadas por células sanas idénticas a las que sustituyen. Los cuatro principales tratamientos más utilizados son: plasma rico en plaquetas (PRP), aspirado y concentrado de médula ósea (BMAC) y aspirado y concentrado de tejido adiposo, y la aplicación de células madre. Estos tratamientos biológicos mimetizan las vías naturales de curación de lesiones al llevar al sitio de lesión las moléculas y proteínas bioactivas necesarias para la regeneración y reparación de los tejidos a tratar. ${ }^{1}$ Cada año se realizan más de dos millones de trasplantes de hueso debido a lesiones traumáticas, defectos congénitos o enfermedades; lo que convierte al hueso en el segundo tejido más trasplantado en todo el mundo. Los mismos principios que se aplican para el tejido óseo se aplican para los ortobiológicos. Los tratamientos ortobiológicos pueden realizarse en tres escenarios: 1) consultorio; 2) gabinete de rayos $X$ y 3 ) quirófano. Aquéllos que se pueden efectuar en consultorio son aproximadamente $70 \%$ incluyendo los que se hacen guiados por ultrasonido. El resto requiere de fluoroscopio y/o quirófano. Lo que se aconseja en estos tiempos de pandemia es diferir todos lo programados y electivos. Los primeros se podrán seguir efectuando en aquellos pacientes que no hayan mostrado respuesta al manejo conservador o que éste haya servido como método para posponer cirugía electiva durante la contingencia. De ser necesario realizar un procedimiento de infiltración se deberán seguir todos los procedimientos recomendados durante la contingencia de COVID-19. Se aconseja hacer las pruebas diagnósticas necesarias tres días antes de su procedimiento. ${ }^{2}$ 


\section{RECOMENDACIONES:}

1. Los pacientes que requieran de un procedimiento ortobiológico deben ser reprogramados y manejados con medicamentos, ya que no es un procedimiento de prioridad. Lo anterior con base en las recomendaciones de organizaciones como FEMECOT, AAOS, AOSM, AO, OMS; entre otras. ${ }^{3,4}$

2. En aquellos pacientes en quienes se requiera un procedimiento quirúrgico para control de dolor y cuando exista un alto riesgo de que el paciente operado presente neumonía, los ortobiológicos son una mejor opción que la cirugía.

3. En caso de ser necesario realizar un procedimiento se deberán seguir los protocolos ya descritos. Considerar a todo paciente como COVID-19 positivo, a menos que se tenga una prueba reciente negativa. ${ }^{5}$

4. Se deberá tomar la temperatura al paciente, proveerlo de cubrebocas, una bata y guantes. No permitir el acceso a familiares a menos que se trate de una persona mayor o que requiera de asistencia de un cuidador.

5. Uso de careta, goggles y cubrebocas del personal del consultorio médico. Quien haga la extracción de la sangre, preparación de material biológico y la infiltración, deberá además de lo anterior portar cubrebocas N95, cubrezapatos impermeables, bata quirúrgica impermeable y dos pares de guantes.

6. El cirujano que hace la infiltración deberá realizarla sin anestesia o utilizando sólo anestesia local. La anestesia regional también podrá utilizarse en casos seleccionados. En caso de ser un procedimiento guiado por ultrasonido utilizar cubiertas estériles para el transductor y su cable.

7. En caso de requerir uso de quirófano, se debe hacer sin presión de aire: equipo de protección personal (EPP) que incluya cubrebocas N95, batas impermeables y anteojos goggles y/o careta según sea el caso (Figura 1). Evitar uso
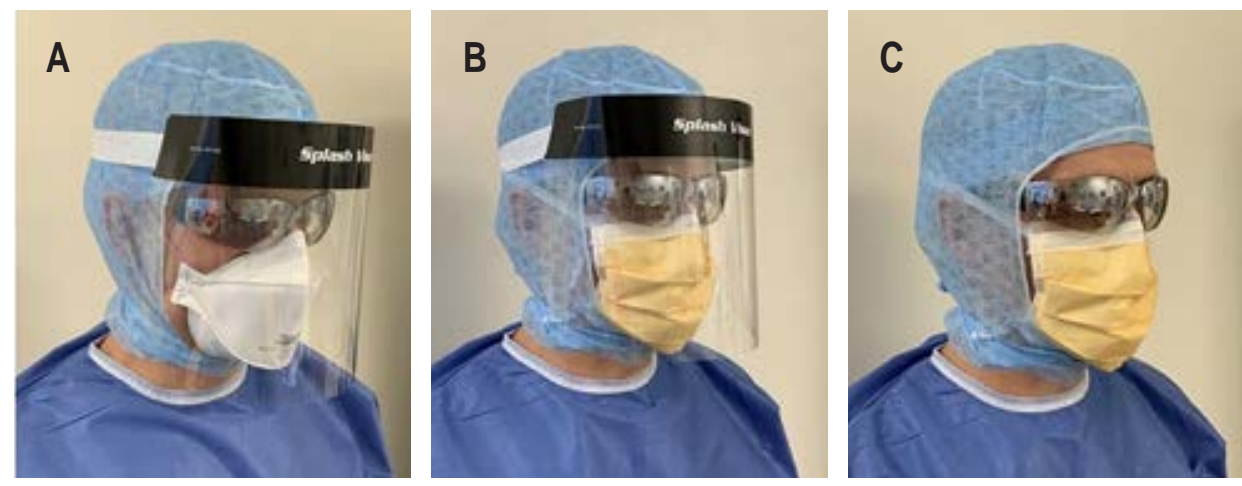

Figura 1: Tipos de protección facial recomendada para realizar procedimientos de ortopedia regenerativa. A) Caso positivo o sospechoso. Mascarilla N95, goggles, gorro completo, careta, bata impermeable y doble par de guantes. B) Caso negativo con riesgo de aerolización. Mascarilla tricapa pegada con cinta adhesiva, el resto parecido al caso anterior. C) Caso negativo con riesgo bajo de aerolización. Sin careta e igual al caso b.

Tomado de: Baldock TE, Bolam SM, Gao R, Zhu MF, Rosenfeldt MP, Young SW, et al. Infection prevention measures for orthopaedic departments during the COVID-19 pandemic: a review of current evidence. Bone \& Joint Open. 2020. Available in: https://doi.org/10.1302/2046-3758.14.BJO-2020-0018.R1. 
de equipo o lavado que produzca aerolización. Si se realizó una incisión, NO utilizar cauterio y hacer el cierre con suturas absorbibles para evitar la visita para retiro de suturas. ${ }^{6}$

8. Dar seguimiento por telemedicina de ser posible y en caso de ser necesaria la presencia física, diferir las consultas a cada cuatro u ocho semanas.

9. Como cirujanos ortopédicos somos el líder de nuestro equipo y debemos garantizar la seguridad de nuestro personal, los pacientes y la de nosotros mismos. Es imperativo que nos familiaricemos con los protocolos de uso de equipo de protección personal (EPP) y recomendaciones mientras luchamos contra esta pandemia de COVID-19. Hasta que exista vacuna o tratamiento, debemos brindar a nuestros pacientes y a todos los participantes de estos procedimientos la mayor protección posible.

\section{BIBLIOGRAFÍA}

1. Padilla S, Sanchez M, Anitua E. Human-based biological and biomimetic autologous therapies for musculoskeletal tissue regeneration. Trends Biotechnol. 2017; 35:192-202.

2. Baldock TE, Bolam SM, Gao R, Zhu MF, Rosenfeldt MP, Young SW, et al. Infection prevention measures for orthopaedic departments during the COVID-19 pandemic: a review of current evidence. Bone \& Joint Open. 2020. Available in: https://doi.org/10.1302/2046-3758.14.BJO-2020-0018.R1.

3. AAOS: Navigating the COVID-19 PANDEMIC-May 2020. https://www.aaos.org/globalassets/about/ covid-19/aaos-clinical-considerations-during-covid-19.pdf?

4. Quintero S, López AL, Pérez GH, Izaguirre HA, Cámara AF, Gutiérrez MI, et al. Declaración de Posición y Recomendaciones FEMECOT Ante la Pandemia COVID-19. FEMECOT YouTube https://youtu.be/ h5IPgOEcfj0.

5. Chang LZ, Ye CM, Sim MA, Lim J, Castañeda P, Green DW, et al. Surgical considerations in patients with COVID-19. What Orthopaedic Surgeons should know. J Bone Joint Surg Am. 2020; 102: e(50. htti://dx.doi.org/10.2106/.JBJS.20.00513.

6. COVID-19: Considerations for Optimum Surgeon Protection Before, During, and After Operation. American College of Surgeons 2020. https://www.facs.org/-/media/files/covid19/considerations_ optimum_surgeon_protection.ashx. 